\title{
Patient delay in reporting oral cancer is poorly understood
}

\author{
What factors influence patient delay in reporting oral cancer?
}

\author{
Scott SE, Grunfeld EA, McGurk M. Patient's delay in oral can- \\ cer: a systematic review. Community Dent Oral Epidemiol 2006; \\ 34:337-43
}

Data sources Studies were sourced using databases as follows: Allied and Complementary Medicine, CANCERLIT, CINAHL, Embase, Medline, and PSYCHINFO. Researchers known to be involved in this area of study were contacted and bibliographic references were manually searched.

Study selection Studies were only included if they were published in the English language; were restricted to participants who had malignancies of the oral cavity; at least $90 \%$ of these patients had a histological diagnosis of squamous cell carcinoma; and if they reported data allowing statistical analysis of the duration of patient delay in reporting their cancer.

Data extraction and synthesis Details of the data extraction process are not given and a qualitative synthesis is presented because of the small number of studies with good methodology and the variability in the factors examined.

Results Of the 148 papers identified as relevant, only eight studies met the inclusion criteria for the review. In these studies, most clinical/ tumour factors, socio-demographic variables, and patient health-related behaviours did not influence the duration of patient delay. Healthcare factors and psychosocial factors may play a role but the research in this area is sparse, atheoretical and of poor quality.

Conclusions Patient delay in reporting oral cancer is a problem and yet, at present, the reasons for such delays are poorly understood and under-researched. Systematic, high-quality and theory-driven research in this area is urgently required.

\section{Commentary}

Early detection of malign ancy is difficult to study: in a review conducted by the Centre for Reviews and Dissemination in York, to inform the National Institute for Health and Clinical Excellence guidance for the NHS in England and Wales (on Improving Outcomes in Head and Neck Cancers; www.nice.org.uk/page.aspx?o=233553), only two studies were identified. These suggested that patients whose cancers were detected "later" (whether defined in relation to an experience of delay in diagnosis or later stage at diagnosis) require more extensive treatment and experience poorer outcomes, and that the majority (58\%) of delays were caused by patients delaying consultation with health professionals. Consequently this review is welcome.

This review has clear inclusion criteria and, although some information is given regarding the selection process, it would have been helpful to know whether selection and data extraction were carried out in duplicate. Restriction to English language studies may also have had an effect on the findings. The fact that no metaanalysis could be carried out is unsurprising given the topic under consideration and the variation in the factors that were considered in the included studies. Consequently the results are unsurprising and the urgent call for high-quality theory-driven research in the area should be supported.

Although there is much new research under way in relation to treatments for oral cancer, and the Cochrane Oral Health Group are undertaking a systematic review of treatments for oral cancer, there remains a lack of awareness of mouth cancer (www.mouthcancerfoundation.org) amongst patients and professionals which may contribute to patient delay. This can be addressed, as the successful West of Scotland Cancer Awareness Programme oral cancer campaign showed. ${ }^{1}$

Delay in seeking treatment also raises issues as to whether a screening programme should be introduced: this has been discussed on several occasions for the UK. The current UK National Screening Committee policy position (www.library.nhs.uk/screening/view Resource.aspx? searchText=Oral+Cancer\&resID=61087) is that a screening programme should not be offered, but that opportunistic screening by all health professionals should be encouraged.

\section{Derek Richards \\ Centre for Evidence-based Dentistry, Oxford, UK}

1. Rodgers ], Macpherson LM. General dental practitioners' perceptions of the West of Scotland Cancer Awareness Programme oral cancer campaign. Br Dent J 2006; 24; 200:693-697.

Evidence-Based Dentistry (2007) 8, 72. doi:10.1038/sj.ebd.6400472

Address for correspondence: Suzanne Scott, Health Psychology Section, Institute of Psychiatry (Guy's), 5th Floor, Thomas Guy House, London SE1 9RT, UK.

E-mail: suzanne.scott@kcl.ac.uk 